\title{
On a Scalable, Non-Blocking Optical Router for Photonic Networks-on-Chip Designs
}

\author{
Xianfang Tan ${ }^{1}$, Mei Yang ${ }^{1}$, Lei Zhang ${ }^{1}$, Yingtao Jiang ${ }^{1}$, Jianyi Yang ${ }^{2}$ \\ ${ }^{1}$ Department of Electrical and Computer Engineering, University of Nevada, Las Vegas, NV, USA \\ ${ }^{2}$ Department of Information Science and Electrical Engineering, Zhejiang University, Hangzhou, China \\ Emails: tanx@unlv.nevada.edu, Mei.Yang@unlv.edu, zhang16@unlv.nevada.edu, yingtao@egr.unlv.edu, yangjy@zju.edu.cn
}

\begin{abstract}
Photonic Networks-on-Chip (NoC) is considered as a promising candidate to interconnect a large number of processing cores. The heart of a photonic NoC is the onchip photonic interconnection network which is composed of silicon waveguides and optical routers. In this paper, we propose a scalable and non-blocking passive optical router design using micro-ring resonators (MRRs), namely the generic wavelength-routed optical router (GWOR). We first introduce a $4 \times 4$ GWOR router structure and then show how to construct larger size GWORs using $4 \times 4$ GWOR as the basic building block. The wavelength assignment schemes for any size GWORs are also derived. For $N \times N$ GWOR, $N$-1 input wavelengths are needed and $\mathrm{N}$-2 different types of MRRs are used. The analysis shows that $4 \times 4$ GWOR uses the least number of MMRs and has the least power loss compared with existing router designs.
\end{abstract}

Keywords: Micro-ring resonator, photonic NoC, optical router

\section{INTRODUCTION}

The rapid advance of technology continues to push up transistor integration capacity, which has enabled a large number of processing cores to be crapped into a Chip Multiprocessor (CMP) or a Multiprocessor System-on-Chip (MPSoC) [1]. A vital challenge faced by many-core-based CMPs and MPSoCs is the design of a scalable on-chip communication architecture which can meet the large bandwidth capacities and stringent latency requirements demanded by these many cores in a power-efficient fashion [1]. Networks-on-Chip (NoC) has been widely adopted as the new on-chip communication paradigm with its better scalability [5]. However, the continuously shrinking feature sizes and increasing complexity of future CMPs and MPSoCs have made metallic interconnects a formidable task to continue to satisfy the bandwidth and latency requirements within the on-chip power budget [1][16]. With the potential of delivering performance-per-watt scaling, photonic NoCs have been proposed as a promising alternative to electronic NoC [14]. The recent advances in nanoscale silicon photonics and development of silicon photonic devices [11][4][9][10][12][13] have made photonic NoCs closer to a reality.

The heart of a photonic $\mathrm{NoC}$ is the on-chip photonic interconnection network which is composed of silicon waveguides and optical routers [14]. The basic function of a router is to route signals between a set of input and output ports. An optical router is generally constructed by waveguides and optical switches. Micro-ring resonators (MRR)-based optical switches are typically adopted due to their ultracompact size, simple-mode resonances, and ease of phase- matching between the MRR and its coupled waveguides [11]. The basic operation of a MRR is shown in Fig. 1(a). The input light signal will be coupled through the drop port if the input wavelength $\lambda_{i}$ is the same as one of the resonance wavelengths of the MMR, say, $\lambda_{r}$, i.e., $\lambda_{i}$ satisfies the following equation

$$
m \lambda_{i}=n_{\text {eff }} L \text {, }
$$

where $m$ is an integer, $n_{\text {eff }}$ is the effective index of the optical mode, and $L$ is the length of the resonating cavity [14]. Fig. 1(b) shows a $2 \times 2$ optical switch constructed with two MRRs with the same resonance wavelength. By using different size MRRs or tuning the refractive index through either thermooptic (TO) [17] or electro-optic (EO) effect [14], an incoming signal can be switched to the destined output port based on its wavelength.

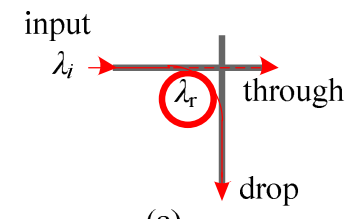

(a)

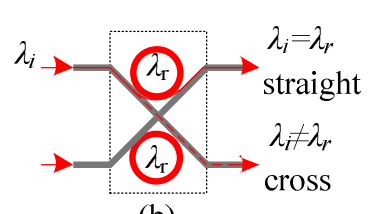

(b)
Figure 1. (a) Basic operation of MRR, and (b) $2 \times 2$ switch.

Optical routers can be classified into two classes according to if TO or EO tuning is used on MMR-based switches: i) active routers using tunable switches, and ii) passive routers with fixed wavelength assignment. Existing non-blocking active router designs include the $5 \times 5$ matrix-based crossbar [15], the optimized $5 \times 5$ crossbar with self-communication disallowed [14], the $4 \times 4$ bidirectional hitless router [17], and the $5 \times 5$ Cygnus router [6]. These active routers can help reduce the number of wavelengths needed, but require extra tuning circuitry for each MRR so that the input light signals can be routed to the destined outputs without confliction. The TO effect can achieve a wavelength tuning range of $20 \mathrm{~nm}$ [9], however, it requires extra heating/cooling time (in microseconds [16]) and higher power consumption $(0.25 \mathrm{~nm} / \mathrm{mW}[17])$. On the other hand, the wavelength tuning range that the EO effect can achieve is limited to $2 \mathrm{~nm}$ [17] with varied driving power (18 105 $\mu \mathrm{W}$ [16]).

As for passive routers, the routing patterns are set at design time. Hence, they do not require extra tuning and control circuits as active routers. Existing non-blocking passive routers include the $\lambda$-router constructed by cascading $2 \times 2$ optical switches [3], the WRON router that has similar structure as $\lambda$ router [18][19], and the $5 \times 5$ passive wavelength-router [8].

In this paper, we focus on passive routers and propose a scalable and non-blocking optical router architecture, namely the generic wavelength-routed optical router (GWOR). The 
wavelength assignment schemes are derived for GWORs. Comparisons of GWOR with matrix-based crossbar, WRON, $\lambda$-router, and hitless router in terms of the number of MRRs used and power loss are also provided.

\section{ROUTER ARCHITECTURE OF $4 \times 4$ GWOR}

Fig. 2 shows the $4 \times 4$ GWOR which has four bidirectional ports located at four directions, north $(\mathrm{N})$, west $(\mathrm{W})$, south $(\mathrm{S})$, and east (E). Two horizontal and two vertical waveguides are used. Each waveguide is dedicated for direct connection between an input-output pair denoted by $I_{\mathrm{i}} \rightarrow O_{3-i}$ (where $i=0,1$, 2 or 3 ). For each waveguide, it has one intersection with each one of the waveguides on the orthogonal direction. As a result, the four waveguides form a basic check shape.

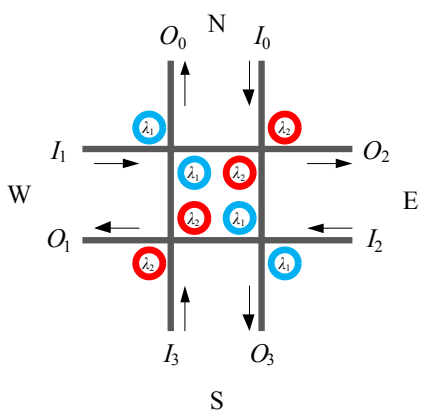

Figure 2. $4 \times 4$ GWOR.

For GWOR, non-blocking routing is realized by assigning MRRs to the appropriate corners of the waveguide intersections so that all input light signals can be directed to their destined output ports. Assume no self-communication is allowed (i.e., input signal from $I_{i}$ will not go to $O_{i}$ ), there exist 12 possible input-output pairs. As four pairs $\left(I_{i} \rightarrow O_{3-i}\right)$ are directly connected by the four waveguides, at least eight MRRs are needed to realize the routing of the rest eight pairs.

The principle of wavelength assignment is to assign the minimal number of wavelengths to the input-output pairs so that any input-output communication can be realized without confliction. Given the input-output pair $\left(I_{i} \rightarrow O_{j}\right)$, the input wavelength $C(i, j)$ is determined by

$$
C(i, j)=\left\{\begin{array}{cc}
- & i=j \\
\lambda_{3} & i+j=3, \quad i \neq j \\
\lambda_{\bmod (2 j, 3)} & i=3, \quad 0<j<3 \\
\lambda_{\bmod (3-2 i, 3)} & j=0, \quad 0<i<3 \\
\lambda_{\bmod (j-i, 3)} & \text { others }
\end{array}\right.
$$

Tab. I lists the wavelength assigned to each input-output pair. To minimize the number of wavelengths, it can be seen from Tab. I that wavelengths are repeatedly used, i.e., $C(i$, $j)=C(3-i, 3-j)$. Hence, the same set of wavelengths is used for all rows and all columns.

TABLE I. ROUTING WAVELENGTH ASSIGNMENT OF THE $4 \times 4$ GWOR

\begin{tabular}{|c|c|c|c|c|}
\hline & $O_{0}$ & $O_{1}$ & $O_{2}$ & $O_{3}$ \\
\hline$I_{0}$ & - & $\lambda_{1}$ & $\lambda_{2}$ & $\lambda_{3}$ \\
\hline$I_{1}$ & $\lambda_{1}$ & - & $\lambda_{3}$ & $\lambda_{2}$ \\
\hline$I_{2}$ & $\lambda_{2}$ & $\lambda_{3}$ & - & $\lambda_{1}$ \\
\hline$I_{3}$ & $\lambda_{3}$ & $\lambda_{2}$ & $\lambda_{1}$ & - \\
\hline
\end{tabular}

Note: - stands for not supported.

According to the wavelength assignment in Tab. I, to route the light signals from $I_{i}$ to $O_{j}$ and from $I_{3-i}$ to $O_{3-j}(i, j=0,1,2$ or 3 , $i \neq j$, and $i+j \neq 3$ ), two identical MRRs corresponding to the assigned input wavelength are placed at the corners of the intersection where the light signals should be directed to their destined output ports. As shown in Fig. 2, only two types of MRRs and totally $8 \mathrm{MRRs}$ are used in the $4 \times 4$ GWOR. It is easy to verify that $4 \times 4$ GWOR is non-blocking for any unicast communication using the input wavelengths assigned in Tab. I. In addition, multicasting and broadcasting can be supported by multiplexing multiple input wavelengths at any input port.

\section{GENERALIZATION OF GWOR}

\section{A. Generalization of GWOR to Even Number of Inputs/Outputs}

An $N \times N$ GWOR (where $N=2 n$ and $n \geq 2$ ) can be built based on the $4 \times 4$ GWOR. $N$ waveguides are used and each waveguide is dedicated for the direct connection between one input-output pair denoted by $I_{i} \rightarrow O_{j}$ (where $i, j=0,1, \ldots, N-1$, and $i+j=N-1$ ), The $N$ waveguides are partitioned into $N / 2$ groups and each group consists of two parallel waveguides. The following rules are followed to interconnect the $N / 2$ groups of waveguides:

1) The first two groups of waveguides are laid out as in the $4 \times 4$ GWOR (Fig. 2). The vertical group consists of two parallel waveguides $I_{0} \rightarrow O_{N-1}$ and $I_{N-1} \rightarrow O_{0}$, while the horizontal group consists of $I_{1} \rightarrow O_{N-2}$ and $I_{N-2} \rightarrow O_{1}$. The intersection of the two waveguide groups forms a basic check shape. Assume the waveguides in $4 \times 4$ GWOR have equal length of $l$ and let $i=2$.

2) Extend all waveguides of the router created so far by $2 l / 3$. For the horizontal waveguide group, bend the east end of each waveguide to south direction and keep the two bended waveguides in parallel. Then extend the bended waveguides to align their bottom ends with the bottom ends of the vertical waveguides.

3) Add the next group of waveguides horizontally to the extended router in Step 2), and have the newly added waveguide group intersect with all existing waveguide groups. The newly added group waveguides are marked as $I_{\mathrm{i}} \rightarrow O_{N-1-\mathrm{i}}$ and $I_{N-1-\mathrm{i}} \rightarrow O_{\mathrm{i}}$. Let $i=i+1$.

4) If there are more waveguide groups to be added, add them one by one following Steps 2) and 3).

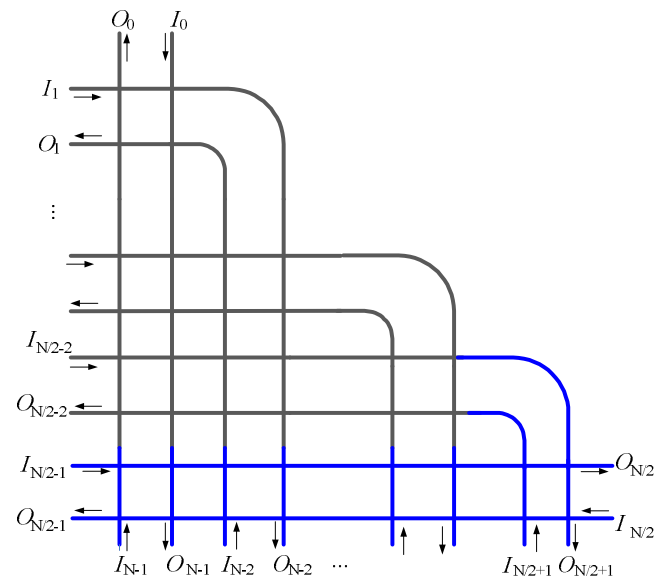

Figure 3. $N \times N$ GWOR built based on $(N-2) \times(N-2)$ GWOR.

Fig. 3 shows how the $N \times N$ GWOR is expanded from the $(N-2) \times(N-2)$ GWOR, where the extension part of vertical waveguides and new added waveguides are colored in blue. 
One can see that any two groups of waveguides form one and only one basic check shape intersection. And there is one and only one intersection between any two orthogonal waveguides.

Similar to the $4 \times 4$ GWOR, the routing of $N \times N$ GWOR is realized by assigning MRRs to the appropriate corners of the waveguide intersections based on the wavelength assignment scheme derived below. Given the input-output pair $I_{i} \rightarrow O_{j}$, the input wavelength $C(i, j)$ is determined by

$$
C(i, j)=\left\{\begin{array}{cc}
- & i=j \\
\lambda_{N-1} & i+j=N-1, \quad i \neq j \\
\lambda_{\bmod (2 j, N-1)} & i=N-1, \quad 0<j<N-1 \\
\lambda_{\bmod (N-1-2 i, N-1)} & j=0, \quad 0<i<N-1 \\
\lambda_{\bmod (j-i, N-1)} & \text { others }
\end{array}\right.
$$

Tab. II lists the wavelength assigned to each input-output pair for $8 \mathrm{x} 8 \mathrm{GWOR}$. According to Tab. II, to route light from input port $I_{i}$ to output port $O_{j}(i, j=0,1, \ldots, N-1)$, an MRR with the assigned wavelength is placed at the only intersection of the waveguides connected to $I_{i}$ and $O_{j}$ when $i$ and $j$ satisfy $i \neq j$ and $i+j \neq N$-1. Fig. 4 shows the constructed $8 \times 8$ GWOR structure.

TABLE II. WAVELENGTH ASSIGNMENT OF $8 \times 8$ GWOR

\begin{tabular}{|c|c|c|c|c|c|c|c|c|}
\hline & $O_{0}$ & $O_{1}$ & $O_{2}$ & $O_{3}$ & $O_{4}$ & $O_{5}$ & $O_{6}$ & $O_{7}$ \\
\hline$I_{0}$ & - & $\lambda_{1}$ & $\lambda_{2}$ & $\lambda_{3}$ & $\lambda_{4}$ & $\lambda_{5}$ & $\lambda_{6}$ & $\lambda_{7}$ \\
\hline$I_{1}$ & $\lambda_{5}$ & - & $\lambda_{1}$ & $\lambda_{2}$ & $\lambda_{3}$ & $\lambda_{4}$ & $\lambda_{7}$ & $\lambda_{6}$ \\
\hline$I_{2}$ & $\lambda_{3}$ & $\lambda_{6}$ & - & $\lambda_{1}$ & $\lambda_{2}$ & $\lambda_{7}$ & $\lambda_{4}$ & $\lambda_{5}$ \\
\hline$I_{3}$ & $\lambda_{1}$ & $\lambda_{5}$ & $\lambda_{6}$ & - & $\lambda_{7}$ & $\lambda_{2}$ & $\lambda_{3}$ & $\lambda_{4}$ \\
\hline$I_{4}$ & $\lambda_{6}$ & $\lambda_{4}$ & $\lambda_{5}$ & $\lambda_{7}$ & - & $\lambda_{1}$ & $\lambda_{2}$ & $\lambda_{3}$ \\
\hline$I_{5}$ & $\lambda_{4}$ & $\lambda_{3}$ & $\lambda_{7}$ & $\lambda_{5}$ & $\lambda_{6}$ & - & $\lambda_{1}$ & $\lambda_{2}$ \\
\hline$I_{6}$ & $\lambda_{2}$ & $\lambda_{7}$ & $\lambda_{3}$ & $\lambda_{4}$ & $\lambda_{5}$ & $\lambda_{6}$ & - & $\lambda_{1}$ \\
\hline$I_{7}$ & $\lambda_{7}$ & $\lambda_{2}$ & $\lambda_{4}$ & $\lambda_{6}$ & $\lambda_{1}$ & $\lambda_{3}$ & $\lambda_{5}$ & - \\
\hline
\end{tabular}

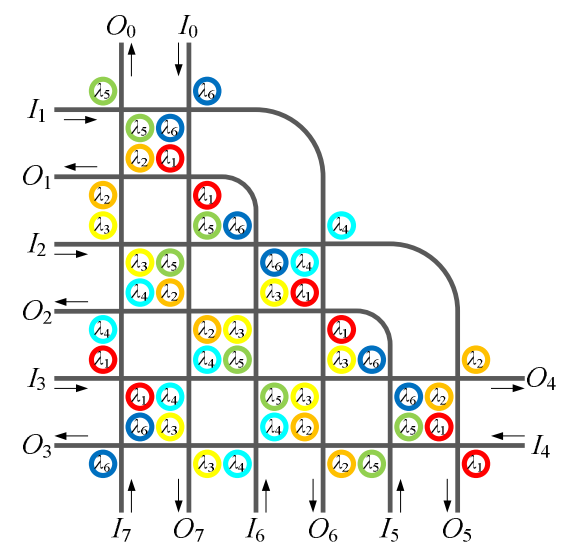

Figure 4. $8 \times 8$ GWOR.

The properties of $N \times N$ GWOR ( $N=2 n$ and $n \geq 2)$ are stated in the following theorems.

Lemma 1: For an $N \times N$ GWOR, the intersections of $N / 2$ waveguide groups form $C_{N / 2}^{2}=N(N-2) / 8$ basic check shapes.

Proof: As shown in the construction process, every two groups form a basic check shape intersection as in Fig. 2. Hence the lemma follows.

Proposition 1: The total number of MRRs used in an $N \times N$ GWOR is $N(N-2)$.

Proof: As in Section 2, 8 MRRs are assigned to each basic check shape intersection. Thus, based on Lemma 1, totally $N(N-2) / 8 \times 8=N(N-2)$ MRRs will be used.
Lemma 2: The number of different types of MRRs used in an $N \times N$ GWOR is $N-2$, corresponding to resonance wavelengths $\lambda_{1}, \lambda_{2}, \ldots, \lambda_{N-2}$.

Proof: Based on Eqn. (3), it is clear that $N-1$ input wavelengths are needed. Hence, $N$-2 different types MRRs are needed because no MRR is used for the input-output pair with direct connection.

Proposition 2: The constructed $N \times N$ GWOR is non-blocking.

Proof: According to the construction process of GWOR, each waveguide has one and only one intersection with each of the two waveguide from the other (N/2-1) groups. Therefore, there are totally $(N-2)$ intersections between one waveguide and other waveguides. At the intersection of the waveguides connected to $I_{i}$ and $O_{j}(i \neq j$ and $i+j \neq N-1)$, two identical MRRs corresponding to the input wavelength of $I_{i} \rightarrow O_{j}$ are assigned, as in Fig. 4. Hence, from any input port, the light signal with the assigned wavelength (from Eqn. (3)) can be directed to the output port connected with the direct waveguide and the other $\mathrm{N}-2$ output ports. In addition, according to the wavelength assignment, on same waveguide, the light signals sent between different input-output pairs (for instance, $I_{0} \rightarrow O_{1}$ and $I_{1} \rightarrow O_{7}$ in Fig. 4) do not conflict with each other because they use different wavelengths. Hence the proposition follows.

\section{B. Generalization of GWOR to Odd Number of Inputs/Outputs}

The method introduced for GWOR with even number of ports can be adapted to build an $N \times N$ GWOR $(N=2 n+1$ and $n \geq 2)$. Here, the $N$ waveguides are divided into $(n+1)$ groups, among which the first $n$ groups are the same as for GWOR with even number of ports, but the $(n+1)_{t h}$ group contains only one waveguide $\left(I_{n} \rightarrow O_{n}\right)$. The interconnection rules in Section 3.A are followed to interconnect the $N$ waveguides. The only difference is at the last step, the intersection of the single waveguide group (i.e., the $(n+1)_{t h}$ group) with any vertical waveguide group only has the two upper crossings of a check shape, which is called reduced check shape.

The wavelength assignment scheme for odd number GWOR is determined by

$$
C(i, j)=\left\{\begin{array}{cc}
- & i=j \\
\lambda_{\bmod (j-i, N)} & \text { others }
\end{array}\right.
$$

TABLE III. WAVELENGTH ASSIGNMENT OF MRR-BASED $5 \times 5$ ROUTER

\begin{tabular}{|c|c|c|c|c|c|}
\hline & $O_{0}$ & $O_{1}$ & $O_{2}$ & $O_{3}$ & $O_{4}$ \\
\hline$I_{0}$ & - & $\lambda_{1}$ & $\lambda_{2}$ & $\lambda_{3}$ & $\lambda_{4}$ \\
\hline$I_{1}$ & $\lambda_{4}$ & - & $\lambda_{1}$ & $\lambda_{2}$ & $\lambda_{3}$ \\
\hline$I_{2}$ & $\lambda_{3}$ & $\lambda_{4}$ & - & $\lambda_{1}$ & $\lambda_{2}$ \\
\hline$I_{3}$ & $\lambda_{2}$ & $\lambda_{3}$ & $\lambda_{4}$ & - & $\lambda_{1}$ \\
\hline$I_{4}$ & $\lambda_{1}$ & $\lambda_{2}$ & $\lambda_{3}$ & $\lambda_{4}$ & - \\
\hline
\end{tabular}

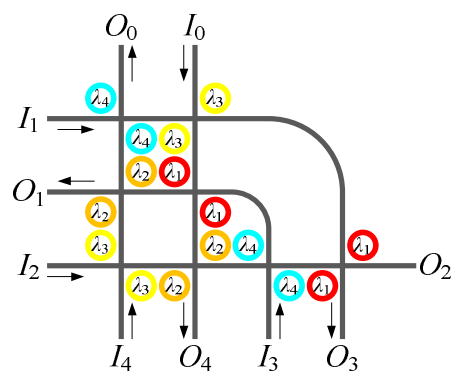

Figure 5. $5 \times 5$ GWOR. 
Tab. III shows the wavelength assignment of $5 \times 5$ GWOR. Similarly, an MRR corresponding to the assigned wavelength is placed at the waveguide intersections to direct light signals from $I_{i}$ to $O_{j}$ when $i$ and $j$ satisfy $i \neq j$ and $i+j \neq N-1$. Fig. 5 shows the constructed $5 \times 5$ router.

The properties of $N \times N$ GWOR $(N=2 n+1$ and $n \geq 2)$ are stated in the following theorems.

Lemma 3: For an $N \times N$ GWOR, the intersections of the $n+1$ waveguide groups form $C_{(N-1) / 2}^{2}=\frac{n(n-1)}{2}$ basic check shapes and $n$ reduced check shape.

Proof: As shown in the construction process, according to Lemma 1, the intersections of the first $n$ group of waveguides form $C_{n}^{2}=C_{(N-1) / 2}^{2}=\frac{n(n-1)}{2}$ basic check shapes and the intersections of the $(n+1)_{t h}$ group with the first $n$ groups form $n$ reduced check shapes.

Proposition 3: The total number of MRRs used in an $N \times N$ GWOR is $(N-1)^{2}$.

Proof: The Proof is similar to that for Proposition 1.

Lemma 4: The total number of different types of MRRs used in an $N \times N$ GWOR is $N$-1, corresponding to wavelengths $\lambda_{1}, \lambda_{2}$, $\ldots, \lambda_{N-1}$.

Proof: On the intersections formed by waveguide $\left(I_{n} \rightarrow O_{n}\right)$ with other waveguides, $N$-1 different types of MRRs are needed.

Proposition 4: The constructed $N \times N$ GWOR is non-blocking. The Proof is similar to that for Proposition 2.

\section{COMPARISON AND ANALYSIS}

In this section, we analyze and compare the number of MRRs used and power loss of the $4 \times 4$ GWOR with that of the existing router designs including the matrix-based crossbar [15], the reduced crossbar [14], hitless router [17], WRON [18][19], and $\lambda$-router [3].

TABLE IV. NUMBER OF MMRS USED IN DIFFERENT $4 \times 4$ ROUTERS

\begin{tabular}{|l|c|c|c|c|c|c|}
\hline & Crossbar & Reduced Crossbar & Hitless Router & WRON $\lambda$-router & GWOR \\
\hline MRRs & 16 & 12 & 8 & 12 & 12 & 8 \\
\hline
\end{tabular}

TABLE V. POWER LOSS OF $4 \times 4$ ROUTERS

\begin{tabular}{|l|c|c|c|c|c|c|}
\hline & Crossbar & $\begin{array}{c}\text { Reduced } \\
\text { Crossbar }\end{array}$ & $\begin{array}{c}\text { Hitless } \\
\text { Router }\end{array}$ & WRON & $\lambda$-router & GWOR \\
\hline Max. Loss (dB) & 1.85 & 1.84 & 1.733 & 1.71 & 1.71 & 1.64 \\
\hline Avg. Loss (dB) & 1.670 & 1.669 & 1.162 & 1.3 & 1.3 & 1.093 \\
\hline
\end{tabular}

Tab. IV shows that GWOR and the hitless router use the least number of MRRs. Tab. V lists the power loss comparison of the maximum and average power loss experienced from one input port to an output port of these routers. The power loss assumption of MRRs and waveguides in [2] is followed here: each MRR has a drop-loss of $1.5 \mathrm{~dB}$ and a through-loss of $0.01 \mathrm{~dB}$, and the crossing loss and bending loss of waveguides are $0.05 \mathrm{~dB}$ and $0.013 \mathrm{~dB}$, respectively. It can be seen that GWOR has the least maximum power loss and average power loss among the six router designs.

\section{CONCLUSION}

In this paper, we proposed a generic passive non-blocking router architecture, namely GWOR, which is scalable from $4 \times 4$ to any size. Routing in GWORs is realized by adopting different input wavelengths and corresponding MRRs. The wavelength assignment schemes are derived for GWORs with even and odd number of input/output ports. A $N \times N$ GWOR needs $N-1$ input wavelengths, and $N(N-2)$ (for $N=2 n$ ) or $(N-1)^{2}$ (for $N=2 n+1$ ) MRRs for routing. Compared with the existing non-blocking router designs, GWOR uses the least number of MRRs and has the least power loss. In addition, the passive nature of GWORs excludes the power needed to drive the tuning and control circuits of those active routers. These advantages make the proposed GWOR a useful building block for future power-efficient photonic NoCs.

\section{REFERENCES}

[1] S. Assefa, F. Xia, and Y. A. Vlasov, "Reinventing germanium avalanche photodetector for nanophotonic on-chip optical interconnects," Nature, vol. 464, pp. 80-84, Mar. 2010.

[2] C. Batten, A. Joshi, J. Orcutt, et al., "Building manycore processor-toDRAM networks with monolithic silicon photonics," in Proc. $16^{\text {th }}$ IEEE Symp. High Performance Interconnects, 2008, pp. 21-30.

[3] M. Briere, B. Girodias, et al., "System level assessment of an optical NoC in an MPSoC platform," in Proc. Design, Auto. \& Test in Europe Opt. Conf. \& Exhibition, 2007, pp. 1-6.

[4] A. W. Fang, B. R. Koch, et al., "A racetrack mode-locked silicon evanescent laser," Optics Express, vol. 16, no. 2, pp. 1393-1398, 2008.

[5] H. Gu, J, Xu, and W. Zhang, "A low-power fat tree-based optical network-on-chip for multiprocessor system-on-chip," in Proc. Design, Auto. \& Test Europe Conf. \& Exhibition, 2009, pp. 3-8.

[6] H. Gu, K. H. Mo, J. Xu, and W. Zhang, "A low-power_low-cost optical router for optical networks-on-chip in multiprocessor systems-on-chip," in Proc. IEEE Int'l Symp. VLSI (ISVLSI), 2009, pp. 19-24.

[7] ITRS 2009, The International Technology Roadmap for Semiconductors. Available: http://www.itrs.net/Links/2009ITRS/2009Chapters_2009Tables/2009_Interconnect.pdf.

[8] N. Kirman, and J. F. Martinez, "A power-efficient all-optical on-chip interconnect using wavelength-based oblivious routing," $A C M$ SIGARCH Comp. Architect. News, vol. 38, no. 1, pp. 15-28, Mar. 2010.

[9] M. Lipson, "Compact electro-optic modulators on a silicon chip," IEEE J. Select. Topics Quantum Electron, vol. 12, no. 6, pp. 1520-1526, Nov. 2006.

[10] M. Lipson, "Guiding, modulating, and emitting light on siliconchallenges and opportunities," J. Lightwave Technol., vol. 23, no.12, pp. 4222-4238, Dec. 2005.

[11] B. E. Little, S. T. Chu, W. Pan, and Y. Kokubun, "Microring resonator arrays for VLSI photonics," IEEE Photon. Technol. Lett., vol. 12, pp. 323-325, Mar. 2000.

[12] A. Liu, "Announcing the world's first 40G silicon laser modulator," (2007) Intel website. Available: http://blogs.intel.com/research/2007/07/40g_modulator.php

[13] J. Liu, X. Sun, R. C. Aguilera, L. C. Kimerling, and J. Michel, "Ge-on-Si laser operating at room temperature," Opt. Lett., vol. 35, no. 5, pp. 679$681,2010$.

[14] A. W. Poon, F. Xu, and X. Luo, "Cascaded microresonator-based matrix switch for silicon on-chip optical interconnect," Proc. IEEE, vol. 97, no. 7, pp. 1216-1238, Jul. 2009.

[15] A. W. Poon, F. Xu, and X. Luo, "Cascaded active silicon microresonator array cross-connect circuits for WDM networks-on-chip," in Proc. SPIE Int'l Soc. Opt. Eng., 2008.

[16] A. Shacham, K. Bergman, and L. P. Carloni, "Photonic networks-onchip for future generations of chip multiprocessors," IEEE Trans. Computers, vol. 57, no. 9, pp. 1246-1260, Sep. 2008.

[17] N. Sherwood-Droz, H. Wang, et al., "Optical $4 \times 4$ hitless silicon router for optical networks-on-chip (NoC)," Optics Express, vol. 16, no. 20, pp.15915-15922, Sep. 2008.

[18] L. Zhang, M. Yang, Y. Jiang, E. Regentova, and E. Lu, "Generalized wavelength routed optical micronetwork in network-on-chip," in Proc. 18th IASTED Int'l Conf. Parallel and Distributed Comp. and Sys., 2006, pp. 698-703.

[19] L. Zhang, M. Yang, Y. Jiang, and E. Regentova, "Architectures and routing schemes for optical network-on-chips," Comp. \& Elec. Engneering, vol. 35, no. 6, pp. 856-877, Nov. 2009. 